\title{
Counterexample of the Riemann Hypothesis
}

\author{
Frank Vega
}

CopSonic, 1471 Route de Saint-Nauphary 82000 Montauban, France

\begin{abstract}
Under the assumption that the Riemann hypothesis is true, von Koch deduced the improved asymptotic formula $\theta(x)=x+O\left(\sqrt{x} \times \log ^{2} x\right)$, where $\theta(x)$ is the Chebyshev function. We obtain a result which contradicts this asymptotic formula. By contraposition, we deduce that the Riemann hypothesis is false.
\end{abstract}

Keywords: Riemann hypothesis, Nicolas inequality, Chebyshev function, prime numbers 2000 MSC: 11M26, 11A41, 11A25

\section{Introduction}

The Riemann hypothesis is a conjecture that the Riemann zeta function has its zeros only at the negative even integers and complex numbers with real part $\frac{1}{2}$ [1]. The Riemann hypothesis belongs to the David Hilbert's list of 23 unsolved problems [1]. Besides, it is one of the Clay Mathematics Institute's Millennium Prize Problems [1]. This problem has remained unsolved for many years [1]. In mathematics, the Chebyshev function $\theta(x)$ is given by

$$
\theta(x)=\sum_{p \leq x} \log p
$$

where $p \leq x$ means all the prime numbers $p$ that are less than or equal to $x$. Say Nicolas $\left(p_{n}\right)$ holds provided

$$
\prod_{q \leq p_{n}} \frac{q}{q-1}>e^{\gamma} \times \log \theta\left(p_{n}\right) .
$$

The constant $\gamma \approx 0.57721$ is the Euler-Mascheroni constant, log is the natural logarithm, and $p_{n}$ is the $n^{\text {th }}$ prime number. The importance of this property is:

Theorem 1.1. [2], [3]. Nicolas $\left(p_{n}\right)$ holds for all prime numbers $p_{n}>2$ if and only if the Riemann hypothesis is true.

We know the following properties for the Chebyshev function:

Theorem 1.2. [4]. If the Riemann hypothesis holds, then

$$
\theta(x)=x+O\left(\sqrt{x} \times \log ^{2} x\right)
$$

for all $x \geq 10^{8}$.

Email address: vega.frank@gmail.com (Frank Vega) 
Theorem 1.3. [5]. For $2 \leq x \leq 10^{8}$

$$
\theta(x)<x
$$

We also know that

Theorem 1.4. [6]. If the Riemann hypothesis holds, then

$$
\left(\frac{e^{-\gamma}}{\log x} \times \prod_{q \leq x} \frac{q}{q-1}-1\right)<\frac{3 \times \log x+5}{8 \times \pi \times \sqrt{x}}
$$

for all numbers $x \geq 13.1$.

Let's define $H=\gamma-B$ such that $B \approx 0.2614972128$ is the Meissel-Mertens constant [7]. We know from the constant $H$, the following formula:

Theorem 1.5. [8].

$$
\sum_{q}\left(\log \left(\frac{q}{q-1}\right)-\frac{1}{q}\right)=\gamma-B=H .
$$

For $x \geq 2$, the function $u(x)$ is defined as follows

$$
u(x)=\sum_{q>x}\left(\log \left(\frac{q}{q-1}\right)-\frac{1}{q}\right) .
$$

We use the following theorems:

Theorem 1.6. [9]. For $x>-1$ :

$$
\frac{x}{x+1} \leq \log (1+x)
$$

Theorem 1.7. [10]. For $x \geq 1$ :

$$
\log \left(1+\frac{1}{x}\right)<\frac{1}{x+0.4}
$$

Let's define:

$$
\delta(x)=\left(\sum_{q \leq x} \frac{1}{q}-\log \log x-B\right) .
$$

Definition 1.8. We define another function:

$$
\varpi(x)=\left(\sum_{q \leq x} \frac{1}{q}-\log \log \theta(x)-B\right) .
$$

Putting all together yields the proof that the inequality $\varpi(x)>u(x)$ is satisfied for a number $x \geq 3$ if and only if Nicolas $(p)$ holds, where $p$ is the greatest prime number such that $p \leq x$. In this way, we introduce another criterion for the Riemann hypothesis based on the Nicolas criterion and deduce some of its consequences. 


\section{Results}

Theorem 2.1. The Riemann hypothesis is true if and only if the inequality $\varpi(x)>u(x)$ is satisfied for all numbers $x \geq 3$.

Proof. In the paper [3] is defined the function:

$$
f(x)=e^{\gamma} \times(\log \theta(x)) \times \prod_{q \leq x} \frac{q-1}{q} .
$$

We know that $f(x)$ is lesser than 1 when Nicolas $(p)$ holds, where $p$ is the greatest prime number such that $2<p \leq x$. In the same paper, we found that

$$
\log f(x)=U(x)+u(x)
$$

where $U(x)=-\varpi(x)$ [3]. When $f(x)$ is lesser than 1 , then $\log f(x)<0$. Consequently, we obtain that

$$
-\varpi(x)+u(x)<0
$$

which is the same as $\varpi(x)>u(x)$. Therefore, this is a consequence of the theorem 1.1.

Theorem 2.2. If the Riemann hypothesis holds, then

$$
\frac{3 \times \log x+5}{8 \times \pi \times \sqrt{x}+1.2 \times \log x+2}+\frac{\log x}{\log \theta(x)}>1
$$

for all numbers $x \geq 13.1$.

Proof. Under the assumption that the Riemann hypothesis is true, then we would have

$$
\prod_{q \leq x} \frac{q}{q-1}<e^{\gamma} \times \log x \times\left(1+\frac{3 \times \log x+5}{8 \times \pi \times \sqrt{x}}\right)
$$

after of distributing the terms based on the theorem 1.4 for all numbers $x \geq 13.1$. If we apply the logarithm to the both sides of the previous inequality, then we obtain that

$$
\sum_{q \leq x} \log \left(\frac{q}{q-1}\right)<\gamma+\log \log x+\log \left(1+\frac{3 \times \log x+5}{8 \times \pi \times \sqrt{x}}\right) .
$$

That would be equivalent to

$$
\sum_{q \leq x} \frac{1}{q}+\sum_{q \leq x}\left(\log \left(\frac{q}{q-1}\right)-\frac{1}{q}\right)<\gamma+\log \log x+\frac{3 \times \log x+5}{8 \times \pi \times \sqrt{x}+1.2 \times \log x+2}
$$

where we know that

$$
\begin{aligned}
\log \left(1+\frac{3 \times \log x+5}{8 \times \pi \times \sqrt{x}}\right) & <\frac{1}{\frac{8 \times \pi \times \sqrt{x}}{3 \times \log x+5}+0.4} \\
& =\frac{3 \times \log x+5}{8 \times \pi \times \sqrt{x}+0.4 \times(3 \times \log x+5)} \\
& =\frac{3 \times \log x+5}{8 \times \pi \times \sqrt{x}+1.2 \times \log x+2}
\end{aligned}
$$


according to theorem 1.7 since $\frac{8 \times \pi \times \sqrt{x}}{3 \times \log x+5} \geq 1$ for all numbers $x \geq 13.1$. We use the theorem 1.5 to show that

$$
\sum_{q \leq x}\left(\log \left(\frac{q}{q-1}\right)-\frac{1}{q}\right)=H-u(x)
$$

and $\gamma=H+B$. So,

$$
H-u(x)<H+B+\log \log x-\sum_{q \leq x} \frac{1}{q}+\frac{3 \times \log x+5}{8 \times \pi \times \sqrt{x}+1.2 \times \log x+2}
$$

which is the same as

$$
H-u(x)<H-\delta(x)+\frac{3 \times \log x+5}{8 \times \pi \times \sqrt{x}+1.2 \times \log x+2} .
$$

We eliminate the value of $H$ and thus,

$$
-u(x)<-\delta(x)+\frac{3 \times \log x+5}{8 \times \pi \times \sqrt{x}+1.2 \times \log x+2}
$$

which is equal to

$$
u(x)+\frac{3 \times \log x+5}{8 \times \pi \times \sqrt{x}+1.2 \times \log x+2}>\delta(x) .
$$

Under the assumption that the Riemann hypothesis is true, we know from the theorem 2.1 that $\varpi(x)>u(x)$ for all numbers $x \geq 13.1$ and therefore,

$$
\varpi(x)+\frac{3 \times \log x+5}{8 \times \pi \times \sqrt{x}+1.2 \times \log x+2}>\delta(x) .
$$

Hence,

$$
\frac{3 \times \log x+5}{8 \times \pi \times \sqrt{x}+1.2 \times \log x+2}>\log \log \theta(x)-\log \log x .
$$

Suppose that $\theta(x)=\epsilon \times x$ for some constant $\epsilon>1$. Then,

$$
\begin{aligned}
\log \log \theta(x)-\log \log x & =\log \log (\epsilon \times x)-\log \log x \\
& =\log (\log x+\log \epsilon)-\log \log x \\
& =\log \left(\log x \times\left(1+\frac{\log \epsilon}{\log x}\right)\right)-\log \log x \\
& =\log \log x+\log \left(1+\frac{\log \epsilon}{\log x}\right)-\log \log x \\
& =\log \left(1+\frac{\log \epsilon}{\log x}\right) .
\end{aligned}
$$

In addition, we know that

$$
\log \left(1+\frac{\log \epsilon}{\log x}\right) \geq \frac{\log \epsilon}{\log \theta(x)}
$$

using the theorem 1.6 since $\frac{\log \epsilon}{\log x}>-1$ when $\epsilon>1$. Certainly, we will have that

$$
\log \left(1+\frac{\log \epsilon}{\log x}\right) \geq \frac{\frac{\log \epsilon}{\log x}}{\frac{\log \epsilon}{\log x}+1}=\frac{\log \epsilon}{\log \epsilon+\log x}=\frac{\log \epsilon}{\log \theta(x)} .
$$


Thus,

$$
\frac{3 \times \log x+5}{8 \times \pi \times \sqrt{x}+1.2 \times \log x+2}>\frac{\log \epsilon}{\log \theta(x)} .
$$

If we add the following value of $\frac{\log x}{\log \theta(x)}$ to the both sides of the inequality, then

$$
\begin{aligned}
\frac{3 \times \log x+5}{8 \times \pi \times \sqrt{x}+1.2 \times \log x+2}+\frac{\log x}{\log \theta(x)} & >\frac{\log \epsilon}{\log \theta(x)}+\frac{\log x}{\log \theta(x)} \\
& =\frac{\log \epsilon+\log x}{\log \theta(x)} \\
& =\frac{\log \theta(x)}{\log \theta(x)} \\
& =1 .
\end{aligned}
$$

We know this inequality is satisfied when $0<\epsilon \leq 1$ since we would obtain that $\frac{\log x}{\log \theta(x)} \geq 1$. Therefore, the proof is done.

Theorem 2.3. The Riemann hypothesis is false.

Proof. If the Riemann hypothesis holds, then

$$
\theta(x)=x+O\left(\sqrt{x} \times \log ^{2} x\right)
$$

for all $x \geq 10^{8}$ due to the theorem 1.2. Now, suppose there is a real number $x \geq 10^{8}$ such that $\theta(x)>x+\sqrt{x} \times \log ^{1.9} x$. That would be equivalent to

$$
\log \theta(x)>\log \left(x+\sqrt{x} \times \log ^{1.9} x\right)
$$

and so,

$$
\frac{1}{\log \theta(x)}<\frac{1}{\log \left(x+\sqrt{x} \times \log ^{1.9} x\right)}
$$

for all numbers $x \geq 10^{8}$. Hence,

$$
\frac{\log x}{\log \theta(x)}<\frac{\log x}{\log \left(x+\sqrt{x} \times \log ^{1.9} x\right)} .
$$

If the Riemann hypothesis holds, then

$$
\frac{3 \times \log x+5}{8 \times \pi \times \sqrt{x}+1.2 \times \log x+2}+\frac{\log x}{\log \left(x+\sqrt{x} \times \log ^{1.9} x\right)}>1
$$

for those values of $x$ that complies with

$$
\theta(x)>x+\sqrt{x} \times \log ^{1.9} x
$$

due to the theorem 2.2. By contraposition, if there exists some number $y \geq 10^{8}$ such that for all $x \geq y$ the inequality

$$
\frac{3 \times \log x+5}{8 \times \pi \times \sqrt{x}+1.2 \times \log x+2}+\frac{\log x}{\log \left(x+\sqrt{x} \times \log ^{1.9} x\right)} \leq 1
$$


is satisfied, then the Riemann hypothesis should be false. Let's define the function

$$
v(x)=\frac{3 \times \log x+5}{8 \times \pi \times \sqrt{x}+1.2 \times \log x+2}+\frac{\log x}{\log \left(x+\sqrt{x} \times \log ^{1.9} x\right)}-1 .
$$

The Riemann hypothesis would be false when there exists some number $y \geq 10^{8}$ such that for all $x \geq y$ the inequality $v(x) \leq 0$ is always satisfied. We ignore when $2 \leq x \leq 10^{8}$ since $\theta(x)<x$ according to the theorem 1.3. We know that the function $v(x)$ is monotonically decreasing for every number $x \geq 10^{8}$. The derivative of $v(x)$ is negative for all $x \geq 10^{8}$. The derivative of $v(x)$ is

$$
\begin{aligned}
v^{\prime}(x) \approx & -\left(\frac{0.1875 \times(0.3+\pi \sqrt{x}) \times(1.66667+\log (x))}{x \times(0.25+\pi \times \sqrt{x}+0.15 \times \log (x))^{2}}\right)+\frac{3}{2 \times x+8 \times \pi \times x^{\frac{3}{2}}+1.2 \times x \times \log (x)} \\
& -\left(\frac{\sqrt{x} \times \log (x)+1.9 \times \log ^{1.9}(x)+0.5 \times \log ^{2.9}(x)}{x \times\left(\sqrt{x}+\log ^{1.9}(x)\right) \times \log ^{2}\left(x+\sqrt{x} \times \log ^{1.9}(x)\right)}\right)+\frac{1}{x \times \log \left(x+\sqrt{x} \times \log ^{1.9}(x)\right)} .
\end{aligned}
$$

Indeed, a function $v(x)$ of a real variable $x$ is monotonically decreasing in some interval if the derivative of $v(x)$ is lesser than zero and the function $v(x)$ is continuous over that interval [11]. It is enough to find a value of $y \geq 10^{8}$ such that $v(y) \leq 0$ since for all $x \geq y$ we would have that $v(x) \leq v(y) \leq 0$, because of $v(x)$ is monotonically decreasing. We found the value $y=10^{8}$ complies with $v(y) \leq 0$. In this way, we obtain that $v(x) \leq 0$ for every number $x \geq 10^{8}$. Consequently, under the assumption that the Riemann hypothesis is true, then

$$
\theta(x)<x+\sqrt{x} \times \log ^{1.9} x
$$

for all $x \geq 10^{8}$. However, we know that $O\left(\sqrt{x} \times \log ^{2} x\right) \neq \sqrt{x} \times \log ^{1.9} x$. Hence, this implies that the Riemann hypothesis is false using the theorem 1.2.

\section{References}

[1] P. B. Borwein, S. Choi, B. Rooney, A. Weirathmueller, The Riemann Hypothesis: A Resource for the Afficionado and Virtuoso Alike, Vol. 27, Springer Science \& Business Media, 2008.

[2] J.-L. Nicolas, Petites valeurs de la fonction d'Euler et hypothese de Riemann, Séminaire de Théorie des nombres DPP, Paris 82 (1981) 207-218.

[3] J.-L. Nicolas, Petites valeurs de la fonction d'Euler, Journal of number theory 17 (3) (1983) $375-388$ doi:10.1016/0022-314X(83)90055-0.

[4] H. Von Koch, Sur la distribution des nombres premiers, Acta Mathematica 24 (1) (1901) 159.

[5] J. B. Rosser, L. Schoenfeld, Approximate Formulas for Some Functions of Prime Numbers, Illinois Journal of Mathematics 6 (1) (1962) 64-94. doi:doi:10.1215/ijm/1255631807.

[6] J. B. Rosser, L. Schoenfeld, Sharper Bounds for the Chebyshev Functions $\theta(\mathrm{x})$ and $\psi(\mathrm{x})$, Mathematics of computation (1975) 243-269doi:10.1090/S0025-5718-1975-0457373-7.

[7] F. Mertens, Ein Beitrag zur analytischen Zahlentheorie., J. reine angew. Math. 1874 (78) (1874) 46-62. doi:10.1515/crll.1874.78.46. URL https://doi.org/10.1515/crll.1874.78.46

[8] Y. Choie, N. Lichiardopol, P. Moree, P. Solé, On Robin's criterion for the Riemann hypothesis, Journal de Théorie des Nombres de Bordeaux 19 (2) (2007) 357-372. doi:10.5802/jtnb.591.

[9] L. Kozma, Useful Inequalities, http://www.lkozma.net/inequalities_cheat_sheet/ineq.pdf, accessed on 2022-01-17 (2021).

[10] A. Ghosh, An Asymptotic Formula for the Chebyshev Theta Function, arXiv preprint arXiv:1902.09231.

[11] G. Anderson, M. Vamanamurthy, M. Vuorinen, Monotonicity Rules in Calculus, The American Mathematical Monthly 113 (9) (2006) 805-816. doi:10.1080/00029890.2006.11920367. 